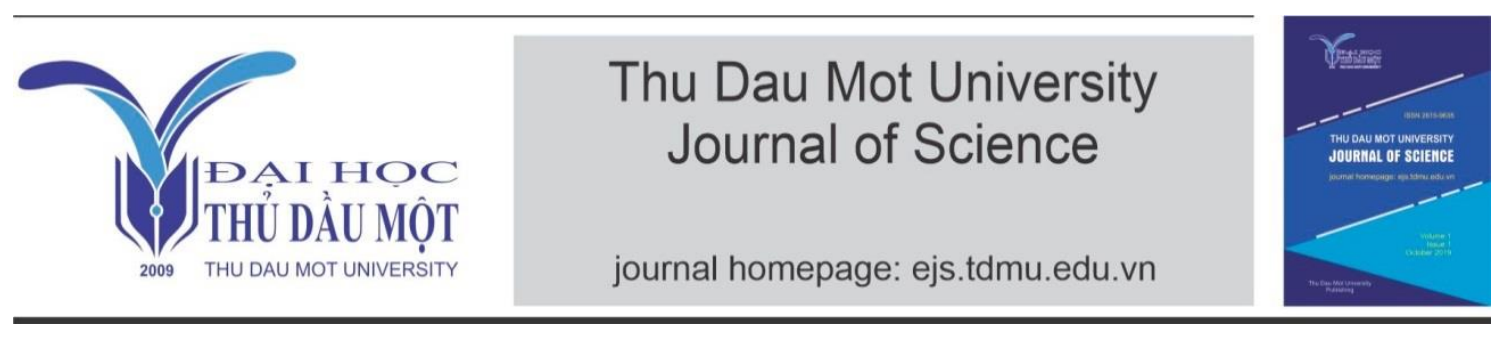

\title{
Prediction of COVID-19 evolution in Binh Duong province using SIR epidemiological model
}

By Pham Bao Quoc (National Cheng Kung University Taiwan), Vo Van On, Nguyen Duy Khanh, Nguyen Thi Lien Thuong, Huynh Thi Phuong Thuy, Hoang Van Ngoc, Nguyen Thanh Tung (Thu Dau Mot University)

Vo Hoang An (Binh Duong Province General Hospital).

Article Info: $\quad$ Received, 30 April 2021, Accepted 2 Sep 2021, Available online 15 Sep 2021

Corresponding author: onvv@tdmu.edu.vn; khanhnd@tdmu.edu.vn

https://doi.org/10.37550/tdmu. EJS/2021.03.219

\begin{abstract}
With the fast growth of the COVID-19 pandemic, in-silico studies based on the susceptible-infected-removed (SIR) epidemiological model are very critical to provide reliable predictions of the COVID-19 evolution that can effectively support governments to issue the right measures to prevent and control the pandemic. In this study, the evolution of the COVID-19 in the Binh Duong province is investigated using the SIR model implemented in R-Studio software, in which the homemade computer codes based on the SIR model are developed using $R$ language to automatically detect the optimal parameters in the model, including the reproduction ratio $R_{0}$, the infection coefficient $\beta$, and the recovery coefficient $\gamma$. The SIR predictions indicate that the number of new positive cases per day in the Binh Duong province is only 40 new cases by November 30, 2021, and the total number of new cases per day becomes zero by middle February 2021. Besides, the Binh Duong province only has 1126 infected cases by November 30, 2021, which reduces to 203 cases at the end of December 2021. Through the SIR results, the COVID-19 pandemic in the Binh Duong is predicted to be ended at the end of December 2021.
\end{abstract}

Keywords: Binh Duong province, COVID-19, SIR model

\section{Introduction}

A new strain of virus called SARS-CoV-2 was first detected in Wuhan city, Hubei 
province, China in December 2019 (Lau et al., 2020). SARS-CoV-2 causes a severe and possibly fatal respiratory distress syndrome termed COVID-19. The COVID-19 has rapidly spread to many countries around the world and has been identified as a global pandemic by the World Health Organization (WHO) in March 2020. SARS-CoV-2 is constantly changing, creating many new variants, rendering the pandemic evolution more and more complicated with the significantly increased number of newly infected cases around the world. To date, the worrisome variants of SARS-CoV-2 have been detected as Alpha (B.1.1.7), Beta (B.1.351, B.1.351.2, and B.1.351.3), Delta (B.1.617.2, AY.1, AY.2, and AY.3), and Gamma (P.1, P.1.1, and P.1.2) (Abdool Karim et al., 2021), in which the Delta variant was found to be more infectious and faster than the other ones. This Delta variant first appeared in India in March 2021 and quickly became the main variant causing a very serious second wave of the pandemic in this country. In addition, the Delta variant has also become the main variant causing new waves of COVID-19 in many countries. As of September 16, 2021, there have been a total number of 227,281,028 people infected with COVID-19 and 4,673,871 deaths worldwide, of which the countries with the highest number of infections are the USA, India, and Brazil. In Vietnam, the Delta variant has caused the fourth extremely serious COVID-19 wave in the southern regions. The Delta variant was first detected in Ho Chi Minh city with only two cases on May 18, 2021; but with the fast growth, the fourth COVID-19 wave has quickly spread almost whole regions in Vietnam. It should be mentioned that there has been a total number of 641,244 positive cases in Vietnam, in which the regions with the highest confirmed cases are Ho Chi Minh city, Binh Duong province, Dong Nai province, and Long An province.

With the fast growth of the COVID-19 pandemic, modeling studies of the COVID-19 based on in-silico simulation methods are very crucial to provide reliable predictions of the COVID-19 evolution that can support the governments to have the right measures to prevent and control the pandemic. To date, many modeling studies have been performed to predict the evolution of the COVID-19 pandemic. Epidemiological models expressed through dynamic ordinary differential equations established based on the mechanism of diseases transmission are very effective for predicting the evolutions of the COVID-19 pandemic. The most commonly studied epidemiological models are SIR (SusceptibleInfectious-Recovered), SEIR (Susceptible-Exposed-Infectious-Recovered), and ESIR (Extended-Susceptible-Infectious-Recovered) (Ahmetolan et al., 2020; Dos Santos et al., 2021). In addition, neural network and machine learning methods have been used to predict the COVID-19 pandemic (Pal et al., 2020; Gupta et al., 2020). Furthermore, other approaches to predict the evolution of COVID-19 have been studied, including the hybrid artificial-intelligence (AI) model (Zheng et al., 2020), a Gauss error function and Monte Carlo simulations (Ciufolini \& Paolozzi, 2020), and the spatial-temporal model (Aràndiga et al., 2020). 
Binh Duong is a key industrial province of Vietnam that has a large density of workers. Thus, the COVID-19 situation is very complicated and unpredictable. Preventing and controlling the COVID-19 in Binh Duong will play an important role in the economic recovery of Vietnam. In that context, it is very critical to provide modeling predictions of the COVID-19 evolution in the Binh Duong province, in which the predicted results can support the government in taking appropriate measures to minimize the impact of the COVID-19. Thus, the research group of modeling and simulation at the Institute of Applied Technology, Thu Dau Mot University has conducted the in-silico study based on the SIR epidemiological model (Ahmetolan et al., 2020; Dos Santos et al., 2021) to predict the evolution of the COVID-19 in the Binh Duong. The results in this study can support the government of the Binh Duong province to issue appropriate measures to effectively prevent and control the COVID-19.

\section{Computational method}

The epidemiological model of SIR (Susceptible-Infectious-Removed) is used to investigate the evolution of the COVID-19 in Binh Duong province. This SIR model has been widely studied by many research groups to predict the evolution of COVID-19 in various countries (Ahmetolan et al., 2020; Dos Santos et al., 2021).

In this SIR study, we consider the number of susceptible people equal to the entire population of the Binh Duong province. The homemade computer codes based on the SIR model are developed to automatically detect the optimal parameters in the model such as the reproduction ratio $\mathrm{R}_{0}$, the infection coefficient $\beta$, the recovery coefficient $\gamma$. The initial input parameters are taken from the daily data on the number of new confirmed cases, the number of recovered cases, the number of deaths in the Binh Duong province. After finding the appropriate optimal parameters based on the data from July 16 to September 14, 2021, on the portal on the number of COVID-19 infections of the Ministry of Health. The parameterized SIR model is numerically computed to result in predictions of COVID-19 evolution in the Binh Duong.

Conceptually, the SIR model is presented as shown in Figure 1.
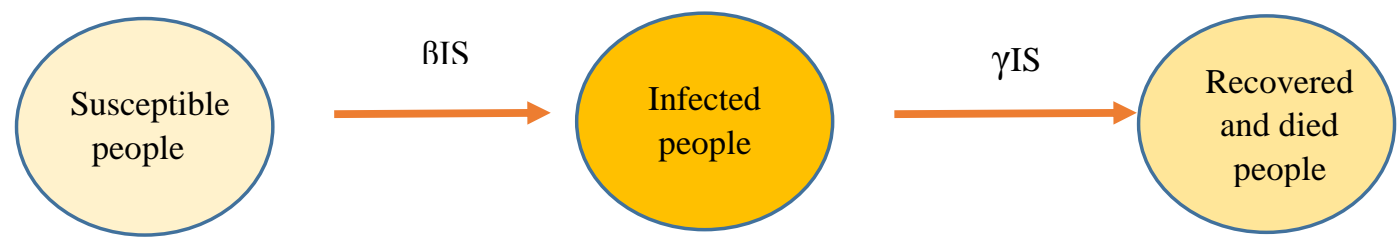

Figure 1. General diagram of the SIR model

Assuming that the natural birth and death rates are not significantly changed during the simulation time of the SIR model. The SIR model is represented by the following differential equations, $\frac{d S}{d t}=-\frac{\beta I S}{N}$

$\frac{d I}{d t}=\frac{\beta I S}{N}-\gamma I$ 
where $\mathrm{S}(\mathrm{t})$ is the number of people at risk of getting the disease at time $\mathrm{t}, \mathrm{I}(\mathrm{t})$ is the number of people infected at time $t, R(t)$ is the total number of people who have recovered and died in time $\mathrm{t}, \mathrm{N}=\mathrm{S}(\mathrm{t})+\mathrm{I}(\mathrm{t})+\mathrm{R}(\mathrm{t})$. The $\gamma$ is the recovery coefficient and $\beta$ is the transmission coefficient which also depends on time.

Three parameters of $S, \gamma$, and $\beta$ have an important influence on the results of the model, so they have been programmed by $\mathrm{R}$ language to automatically optimize with the objective function to minimize the error between the simulation results and the actual results of the number of people infected (I) and the number of people recovered + the number of deaths $(\mathrm{R})$.

Determining these parameters is very difficult because it depends on the different behavioral characteristics of each region. In this study, we set the initial condition of S $\left(\mathrm{S}_{0}\right)$ equal to the current population of the Binh Duong province and used the algorithm to reduce $\mathrm{S}_{0}$ gradually until achieving its probable level ( $\mathrm{S}=297000$ people).

The coefficients of $\gamma$ and $\beta$ are set in the ranges [0.09-0.1] and [0-1], respectively. The results are consistent with actual data showing that the appropriate coefficients $\gamma$ and $\beta$ are 0.09 and 0.185 . After adjusting the SIR model with appropriate parameters, the adjusted SIR model is simulated using R-Studio software to result in the COVID-19 evolution in Binh Duong province.

\section{Results and Discussions}

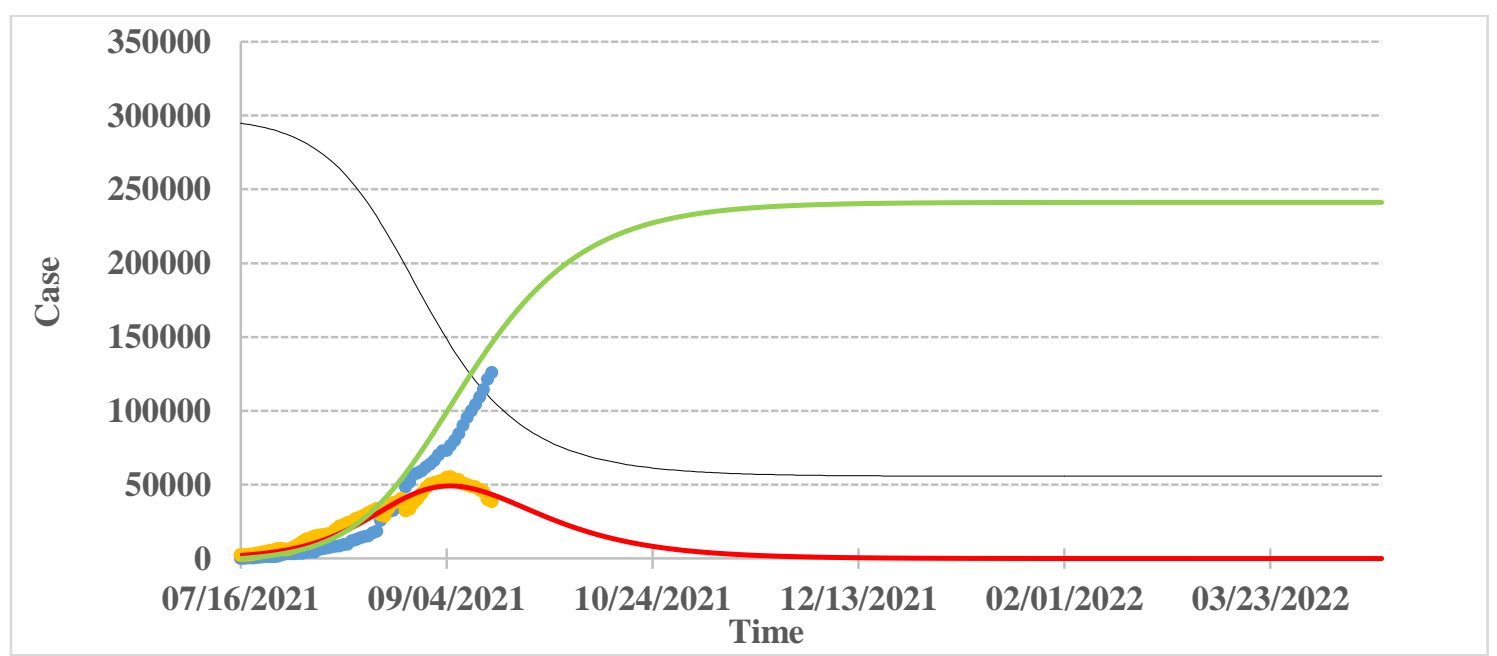

Figure 2. SIR-based results of the COVID-19 evolution in the Binh Duong province

In Figure 2, the black curve shows the number of people susceptible to COVID-19 by simulation, the green curve illustrates the total number of recoveries and deaths by simulation, the red curve displays the new positive cases by day simulation, the orange dashed curve shows the actual number of infections, and the blue dashed curve shows the number of cured and died cases in actual days. 
TABLE 1. Evolution of COVID-19 over several time points

\begin{tabular}{|c|c|c|c|}
\hline Time & New cases & Infected cases & Total infected cases \\
\hline $30-09-2021$ & 1299 & 26146 & 219297 \\
\hline $15-10-2021$ & 543 & 13072 & 231934 \\
\hline $31-10-2021$ & 217 & 5729 & 237467 \\
\hline $15-11-2021$ & 93 & 2557 & 239613 \\
\hline $30-11-2021$ & 40 & 1126 & 240539 \\
\hline $15-12-2021$ & 17 & 492 & 240941 \\
\hline $31-12-2021$ & 7 & 203 & 241123 \\
\hline $18-02-2022$ & 0 & 13 & 241242 \\
\hline
\end{tabular}

Figure 3. Evolution of new positive cases per day according to the SIR model in Binh Duong

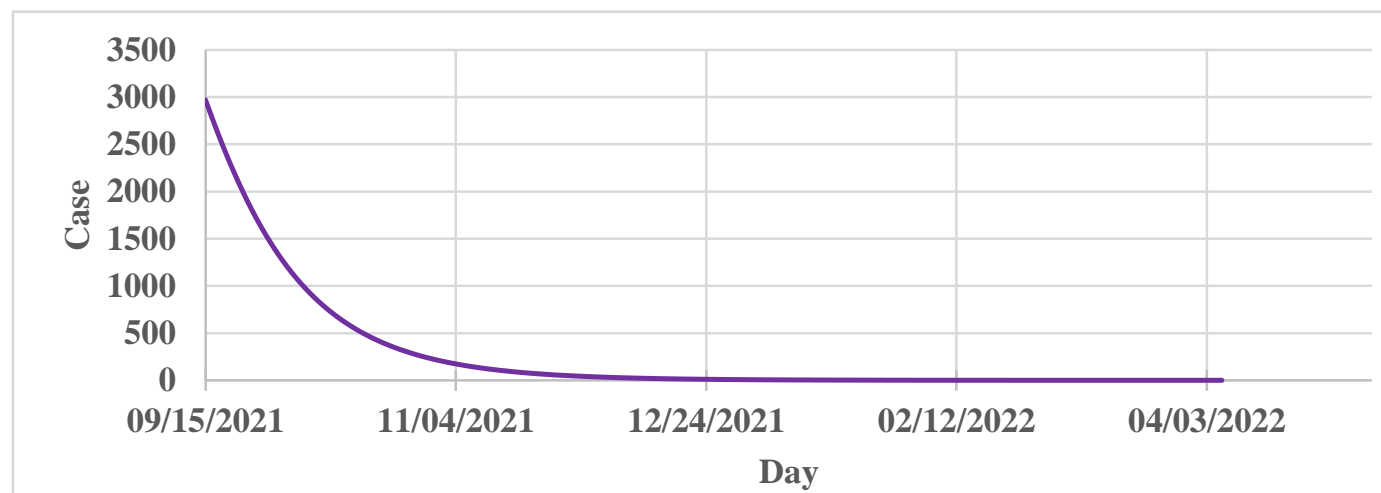

In Figure 3, the purple curve shows the evolution of new cases according to the SIR model prediction in Binh Duong province from September 15, 2021, onward. According to this prediction (see Table 1), by November 30, 2021, the number of new positive cases in the Binh Duong province is only 40 new cases per day, which can be considered as the province has controlled the fourth wave of the COVID-19. As a result, the number of new cases becomes zero by mid-February 2021 .

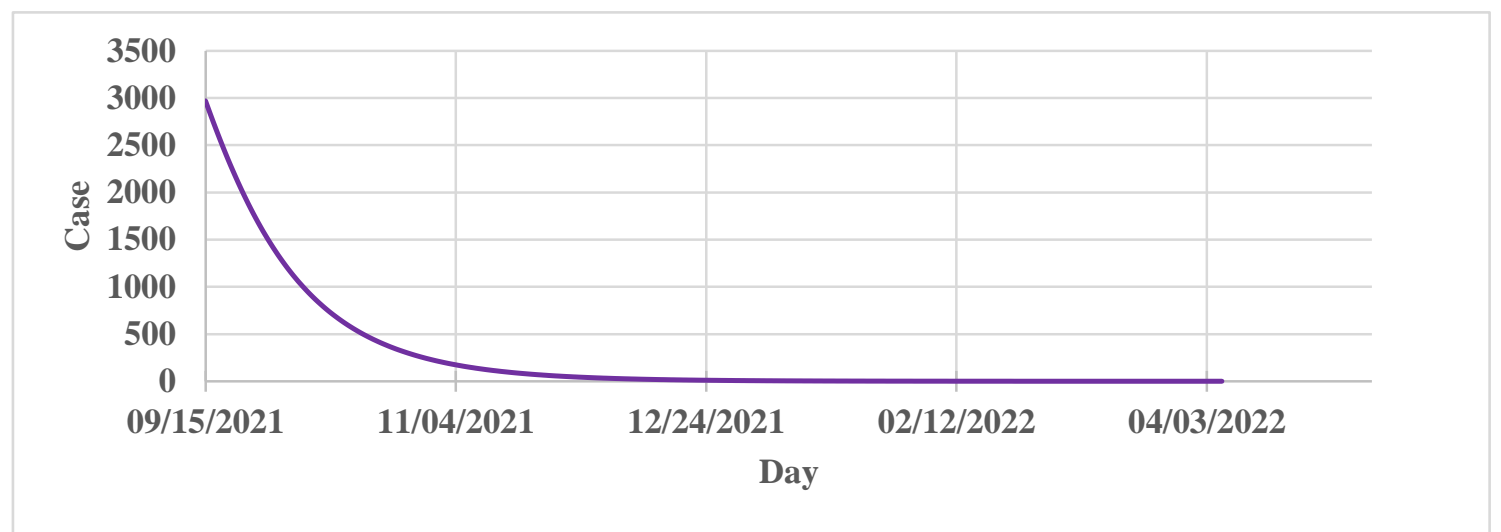

Figure 4. Evolution of the number of infected people according to the SIR model in Binh Duong 
In Figure 4, the red curve shows the number of active cases from September 15, 2021, onward. According to this prediction (see Table 1), as of November 30, 2021, Binh Duong province still has 1126 cases. By the end of December 2021, about 203 cases are still being treated.

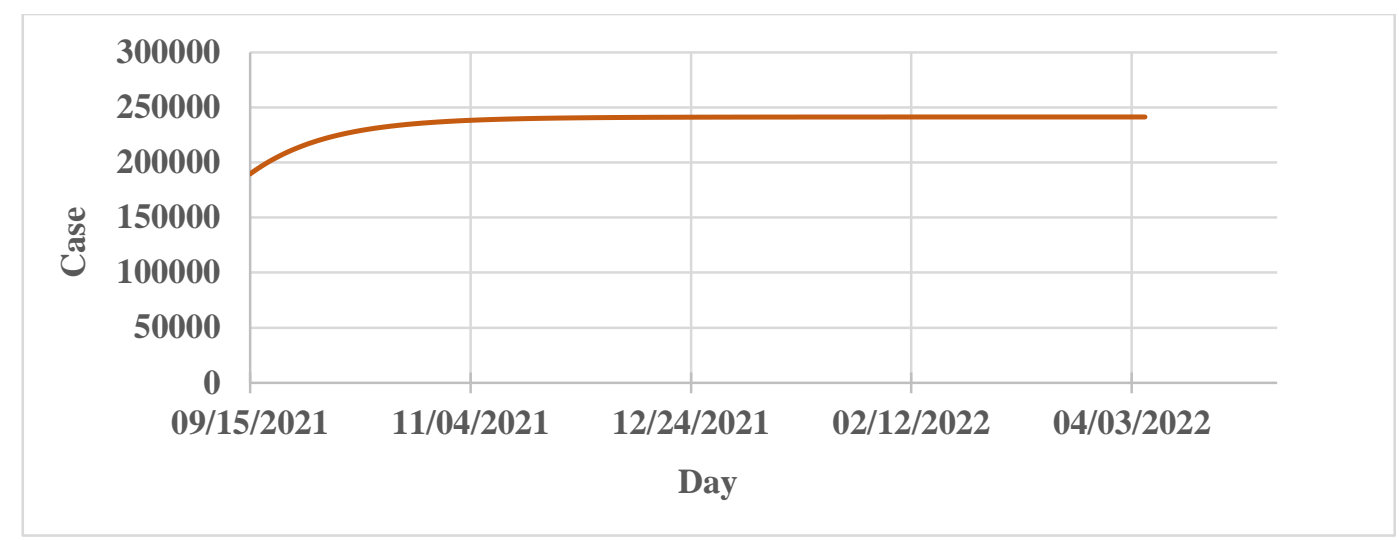

Figure 5. Evolution of the total infected cases according to the SIR model in Binh Duong

In Figure 5, the gray curve shows the evolution of the total number of infected cases since the beginning of the pandemic in the Binh Duong province. According to this prediction, from December 31, 2021 (see Table 1), the total number of infected cases is almost unchanged, as the number of new cases rapidly decreases to zero.

However, in these SIR results, there are still relative errors since the data on new cases, recovered cases, deaths, and total daily infected cases at the early stage of the COVID19 have not yet been updated on time, causing that the simulation results still have large errors. At the later stage of the COVID-19 pandemic, the data of COVID-19 has been updated on time that is closer to the actual epidemiological data. Thus, the error of SIR results in the simulation is reduced at the later stage. Specifically, the average relative error from July 17 to September 15,2021 , is about $26.5 \%$. The relative error of this SIR model is similar to that of other forecasting models, so it can be highly feasible.

\section{Conclusion}

The presented SIR results are highly feasible if there are no other unpredictable changes such as (i) Virus strains create new variants with variable spreading rates; (ii) The disease management policies of the local government have changed a lot (such as policies on isolation, blockade, $\mathrm{F}_{0}$ isolation, treatment, and opening the economy); (iii) Infected cases or uninfected people in other provinces move into the Binh Duong province. In general, the predicted results will be effective if the conditions on distance, isolation of infections, treatment in the Binh Duong province do not change much 
compared to the period from July to September 2021. If the above conditions are better implemented, the end of the pandemic will be earlier; If the current implementation is not as good, the end of the pandemic will be longer than the predicted results of this SIR epidemiological simulation. In addition, this SIR model does not consider birth and death rates due to other factors. The study also assumes that the number of people who have recovered from infection have immunity and are not infected again.

\section{References}

Abdool Karim, S. S., de Oliveira, T., and Loots, G. (2021). Appropriate names for COVID-19 variants. Science, 371, 1215-1215.

Ahmetolan, S., Bilge, A. H., Demirci, A., Peker-Dobie, A, and Ergonul, O. (2020). What can we estimate from fatality and infectious case data using the susceptible-infected-removed (SIR) model? A case study of Covid-19 pandemic. Frontiers in Medicine, 7, 570.

Aràndiga, F et al., (2020). A spatial-temporal model for the evolution of the COVID-19 pandemic in Spain including mobility. Mathematics, 8, 1677.

Arifin, W. N., Chan, W. H., Amaran, S, and Musa, K. I. (2020). A Susceptible-InfectedRemoved (SIR) model of COVID-19 epidemic trend in Malaysia under Movement Control Order (MCO) using a data fitting approach. MedRxiv.

Ciufolini, I, and Paolozzi, A. (2020). Mathematical prediction of the time evolution of the COVID-19 pandemic in Italy by a Gauss error function and Monte Carlo simulations. The European Physical Journal Plus, 135, 1-8.

Cooper, I., Mondal, A., and Antonopoulos, C. G. (2020). A SIR model assumption for the spread of COVID-19 in different communities. Chaos, Solitons \& Fractals, 139, 110057.

Dos Santos, I. F. F., Almeida, G. M. A., and de Moura, F. A. B. F. (2021). Adaptive SIR model for propagation of SARS-CoV-2 in Brazil. Physica A: Statistical Mechanics and its Applications, 569, 125773.

Gupta, R., Pandey, G., Chaudhary, P, and Pal, S. K. (2020). Machine learning models for government to predict COVID-19 outbreak. Digital Government: Research and Practice, 1, 1-6.

Lau, H., Khosrawipour, V., Kocbach, P., Mikolajczyk, A., Schubert, J., Bania, J, and Khosrawipour, T. (2020). The positive impact of lockdown in Wuhan on containing the COVID-19 outbreak in China. Journal of Travel Medicine, 27, taaa037.

Neves, A. G., and Guerrero, G. (2020). Predicting the evolution of the COVID-19 epidemic with the A-SIR model: Lombardy, Italy and Sao Paulo state, Brazil. Physica D: Nonlinear Phenomena, 413, 132693.

Pal, R., Sekh, A. A., Kar, S, and Prasad, D. K. (2020). Neural network-based country-wise risk prediction of COVID-19. Applied Sciences, 10, 6448.

Singh, A. K., Mehra, M., and Gulyani, S. (2021). A modified variable-order fractional SIR model to predict the spread of COVID-19 in India. Mathematical Methods in the Applied Sciences.

Wan Nor Arifin, Weng Howe Chan, Safiya Amaran, Kamarul Imran Musa (2020). A Susceptible-Infected-Removed (SIR) model of COVID-19 epidemic trend in Malaysia 
under Movement Control Order (MCO) using a data fitting approach, medRxiv preprint, doi: https://doi.org/10.1101/2020.05.01.20084384.

Wangping, J., et al., (2020). Extended SIR prediction of the epidemics trend of COVID-19 in Italy and compared with Hunan, China. Frontiers in Medicine, 7, 169.

Zheng, N et al., (2020). Predicting COVID-19 in China using hybrid AI model. IEEE Transactions on Cybernetics, 50, 2891-2904.

https://www.who.int (WHO Director-General's opening remarks at the media briefing on COVID-19 - 11 March 2020).

https://www.sciencemediacentre.org.

https://www.worldometers.info/coronavirus.

https://covid19.gov.vn. 